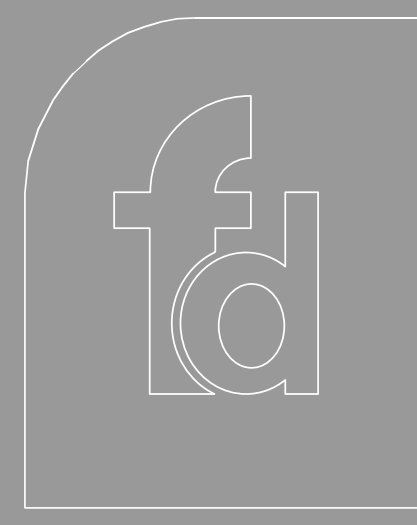

CONTENTS

Literature ovenview

The choice of epidemiological model

Estimating \& valuing health outcomes

Costestimation

Conclusion

Expertopinion

Five-yearview

Key issues

References

Affiliations

${ }^{\dagger}$ Author for correspondence $N$ ational Centre for Immunization Research and Surveillance, University of Sydney and Centre for $\mathrm{H}$ ealth Economics Research and Evaluation, University of Technology at Sydney, Australia; Centre for the Evaluation of Vaccination, University of Antwerp, Belgium

\section{KEYWORDS:}

cost-effectiveness, immunization, modeling

\title{
Methodological issues and new developments in the economic evaluation of vaccines
}

\author{
Philippe Beutels ${ }^{\dagger}$, Eddy Van Doorslaer, Pierre Van Damme and Jane Hall
}

The application of ec onomic evaluation in healthcare, including vaccination programs, has increased exponentially since the 1980s. There are a number of aspects of economic evaluation of vaccine programs that present partic ular challenges to the analyst These include the development of the appropriate epidemiological models from whic $h$ to estimate the costs and benefits, the accurate predic tion of uptake rates; the incorporation of quality adjusted sunvival gains; and the inc lusion of intangible butnonetheless important benefits and costs associated with infectious disease and vac cination. The estimation of marginal intervention costs presents specific difficulties, especially for multivalent vacc ines and valuing costs and benefits overtime is heavily influenced by the choice of disc ountrate, which is still a controversial topic. Developments in the next 5years are likely to address all of these issues and result in more sophisticated and accurate models of vacc ination programs.

Expert Rev. Vaccines 2(5), 649-660 (2003)

The application of economic evaluation in healthcare has increased exponentially since the 1980s. Although the foundations of this were established in the 1960s, pressure on government budgets and the emergence of more and more sophisticated medical technology have encouraged the planners, funders and providers of healthcare to adopt this systematic approach to ensuring value for healthcare expenditure. This is as true in the economic evaluation of vaccination programs (EEVP) as in other parts of the health budget, even though economic evaluation has not been a mandatory component of vaccine introduction as it is has in some countries for new pharmaceuticals [1].

As economic evaluation has become more widely accepted by policymakers, there have been developments in the underlying theory and methods, particularly in the conduct of economic evaluations alongside clinical trials. There are, however, a number of aspects of EEVPs that present particular challenges to the analyst, as outlined in a recent consensus statement from a group of heal th economists with experience in EEVP [2]. A much more detailed account of these key issues is available [3]. These are the focus of this paper.
The essence of any economic evaluation is the comparison of benefits and costs. For vaccination programs, immediate benefits in terms of reduced risks of infection and fewer cases, will occur throughout the population and may give rise to complex indirect effects. Consequently, accurate estimates of benefits require the modeling of the underlying infection process and thus the type of epidemiological model used is critical. The estimation of quality adjusted life years (QALYS), requires the use of a quality adjustment as well as the estimation of survival gains. Although the use of QALYs as the benefit measure is not widely established in EEV Ps, this is important for any program targeting infections with long lasting complications. While QALYs are a more sensitive measure than life years gained, there is increasing concern as to whether they encompass all the benefits of vaccination which are important to and valued by consumers. Therefore, there is renewed interest in other measures of benefit, particularly in willingness to pay for vaccination programs.

In EEV Ps, the intervention costs often have a great impact on the cost-effectiveness for 
two reasons, the incremental cost of the new vaccine and the timing. The incremental cost depends on both the cost of the vaccine and the cost of its administration, which in turn depends on how well it fits into any existing program. Vaccination costs occur in the present while the prevention of disease and any offsetting costs of treatment arise in the future. H ence the choice of discount rate can be highly influential. The uptake rate of the vaccination program is not only critical to estimating the costs of the program but also crucial in accurately modeling the effects of the program.

The next section provides a brief overview of published EEVPs. The paper then discusses each of the above issues in turn.

\section{Literature ovenview}

The annual number of publications of economic evaluations is shown in FIGURE 1 (SEE BOX 1 FOR SEARCH STRATEGY). Economic evaluation in general (solid line), economic evaluation in healthcare (dashed line) and economic evaluation of vaccines (bars) all increased. Restricting the focus to Web of Science (as compared with PubM ed, it excludes newsletters and additional popular medical journals not listed in the Citation Indices but contains scientific economic journals) and to articles on vaccination in humans published in the last 10 years (1993-2002), yields 200 analyses. FIGURE 2 shows the biannual distribution of these remaining papers according to the specific pathogen studied. These results are subject to publication bias, related to both wealth and language, exclude many peer-reviewed non-English publications and do not encompass the gray literature i.e., reports for ministries of health and national health councils, as well as reports from institutions such asW orld $\mathrm{H}$ ealth $\mathrm{O}$ rganization, World Bank, U NICEF and M SF. Pharmaceutical companies also perform in house EEVPs focusing on feasibility and marketing potential, in various stages of vaccine development. Nonetheless, our search provides some indication of the distribution of analytic effort.

Most EEVPs were set in wealthy countries. The high frequency of research on some vaccinepreventable diseases [FIGURE 2] is partly due to the existence of multiple target groups (e.g., travelers, healthcare workers, children and military

A search was conducted using both PubMed (i.e., Medline) and Web of Science (limiting to the Scientific Citation Index Expanded and the Social Science Citation Index) using the combined search string (cost OR costs OR benefits OR costeffectiveness OR cost-benefit OR cost-utility OR economic* evaluation*) AND (vaccine* OR immunisation OR immunization NOT animal) for the title only, both with and without the underlined part. This yields the results in Figure 1 (limited to the period 1980-2002). The search for vaccine-related papers yields more results in PubMed than in Web of Science as the former also contains newsletters and additional popular medical journals that are not listed in the Citation Indices.

Box 1. Explanation of web-based search strategies used. personnel for hepatitis A (HAV) and multiple vaccines (e.g., pneumococcal polysaccharide [PSV ] and the new pneumococcal conjugate vaccine [PCV ]), demanding separate analyses in different settings. W hat these vaccines also have in common is that they are expensive compared with most other widely used vaccines and that they are (were) mainly targeted at "low profile' pathogens. In wealthy countries they mainly affected many elderly patients in the short run (PSV, influenza), few adults in the short run (HAV, varicella-zoster [VZV]), few to many adults in the long run (hepatitis $B$ [H BV ]), or generally mild disease in most children (VZV). Furthermore, the population effectiveness of some of these vaccines (particularly PSV and VZV) has been, and still is, under debate. Due to the multitude of studies on these vaccines, comprehensive reviews can help understand the many specific clinical and analytical problems involved and may offer guidance to decision makers. Such recent reviews are available for each of these much-evaluated vaccines [4-8].

In contrast, during the same period, decisions with a very large budget-impact were taken concerning Haemophilus influenzae type $b(\mathrm{H}$ ib) and $M$ eningococcal $C$ ( $M$ en $C$ ) vaccination in several wealthy countries, with a total of only nine and three published evaluations, respectively. The total disease burden from either $\mathrm{H}$ ib or $\mathrm{M}$ enC is not likely to exceed that of some of the above pathogens in many settings. Public support for these programs may be due to the fact that they prevent rare but severe disease in children, the publicity given to a few cases with severe and even fatal consequences (a variant on the so-called rule of rescue [9]), or concern at a new public health threat.

The search identified very few EEV Ps for developing countries, partly due to publication bias but also because of the limited marketability of vaccines in these countries. This is reflected, for instance, by the few evaluations on diseases which have never been - or are no longer - a public health threat in industrialized countries (FIGURE 2, TABLE 1).

It seems that economic evaluation is not yet a standard ingredient of the appraisal process of new vaccine programs, even in industrialized countries. But it is more likely to be employed to add support for programs that lack popular appeal.

\section{The choice of epidemiological model}

EEVPs are, by necessity, usually based on modeling. In most models, the population is made to flow between mutually exclusive compartments of susceptible, infected (and infectious) and immune people according to functions of discrete time ( $t=0,1$, $2, \ldots)$ or differentiable functions of continuous time ( $t \geq 0$ ) $[10,11]$. This approach is deterministic if there is no randomness in the calculations, implying those rates of flow between compartments are based on population averages. In reality, individuals come in whole entities (and cannot be averaged out to fractions) and the spread of an infection can be regarded as a random process. It can be assumed that this process can be simulated adequately with average rates (i.e., in a deterministic model) if the population at risk is large and 
the infection is not close to elimination. H owever, for small populations (e.g., family size, small islands), stochastic models are better suited because they introduce the randomness associated with disease transmission [12]. Indeed, in a population where infectious and susceptible individuals mix, some susceptible persons will be infected while other susceptible persons will not. While we may know that on average in the long run, say $50 \%$ of such contacts lead to successful disease transmission, it may be that purely by chance, particular contacts do not lead to transmission. Events such as this could have far-reaching consequences in small populations or when virus transmission has become generally rare (as for diseases close to elimination). To our knowledge, EEVPs in humans have always been carried out with deterministic models, which seems acceptable in view of the relatively large populations involved (and the fewer data requirements).

\section{Static \& dynamic models}

A further important distinction can be made between socalled 'static' and 'dynamic' models. In a dynamic model, the force of infection (the probability that a susceptible person acquires infection per unit of time) can change with time. As subsequent cohorts are vaccinated, the proportion of infectious people in the total population decreases. Consequently, the force of infection acting on those remaining susceptible declines as well. A dynamic model takes this into account by cyclically recalculating the force of infection. In a static model, the force of infection stays constant (i.e., the force of infection is assumed to be independent of the proportion of infectious people in the population). Parameters in static models are thus only age-dependent and not time-dependent, as in dynamic models. Static models usually calculate costs and effects for a single aging cohort. H owever, in reality, routine vaccination programs are meant to continue indefinitely, impacting on consecutive cohorts. Thus, over time, the risk of infection changes. Once herd immunity (i.e., the indirect protection of susceptible persons in a largely vaccinated population) is achieved, vaccination will more than proportionally reduce the incidence of infection, increase the average age at infection and increase the length of the inter-epidemic period. Vaccination scenarios that ignore herd immunity effects therefore, will tend to overestimate the residual incidence and underestimate the age at infection of residual cases, as well as the inter-epidemic period.

To date, the overwhelming majority of economic evaluations has been based on static models. The first full EEVP to use a dynamic model was published by Lieu and colleagues [13] in 1994, based on a model developed by $\mathrm{H}$ alloran and colleagues [14]. Edmunds and colleagues investigated the impact for a hypothetical airborne infection, by comparing a static cohort model with an age-independent dynamic population model [15]. They found the differences in results to be greatest at moderateto-high immunization levels and lowest at the extremes (effective immunization of 0 and $100 \%$ ). Results were also more similar for higher basic reproduction numbers (i.e, the number of secondary infections caused by one initial infection in a totally susceptible population). A recent overview of these issues is provided elsewhere [45].

D ynamic models include a future stream of costs, i.e., the vaccination of new cohorts as well as benefits, and may there fore be more sensitive to discounting. The combined effect of both discounting and the multiple cohort set-up may be to make particular population and single cohort models yield similar results. Clearly, quantitative comparisons between static and dynamic models for specific infections and vaccination strategies constitute an important unexplored research topic.

\section{When static models can be appropriate}

Static models seem a priori suited to evaluate changes in vaccination coverage if herd immunity does not play an important role (i.e., when the additional effectiveness per additional vaccinee is constant). O ne particular example is an intervention targeted at a particular risk group which is not a core transmitter group of the pathogen. Immunizing such groups

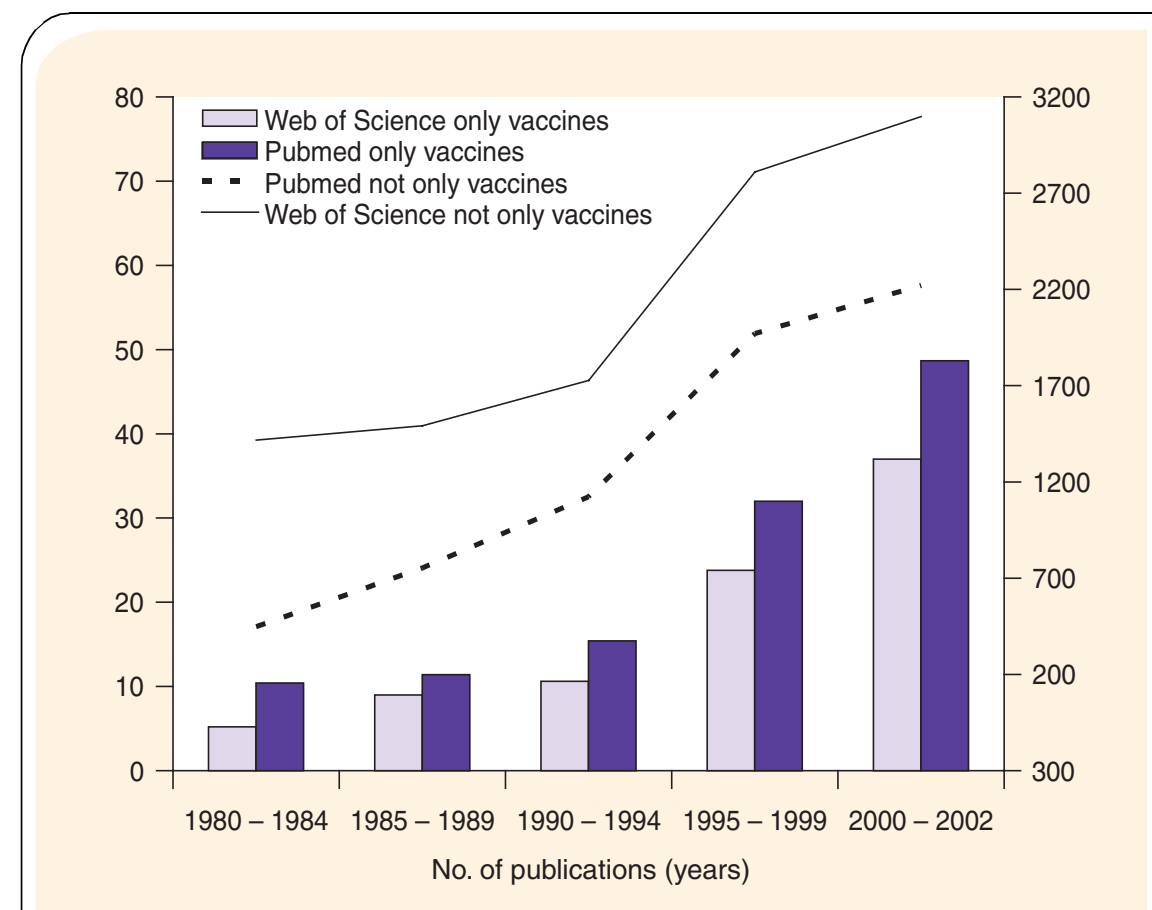

Figure 1. Average annual number of publications related to economic evaluation, retrieved by searching Pubmed and Web of Science (1980-2002). Bars plotted on primary $Y$-axis, lines plotted on secondary Y-axis. 
will not cause intertemporal differences in the transmission of the virus to the groups in question (apart from usually blocking transmission if vaccinated), or in the population as a whole, provided that the number of vaccinees remains relatively small compared with the total population size. Examples of the sort of vaccination programs that fall into this category are H AV vaccination of travelers to high endemic areas, influenza and pneumococcal vaccination programs targeted at the elderly or VZV vaccination of (susceptible) pre-adolescents or heal thcare workers. Another example is where vaccination against an infection will not induce herd immunity, simply because the transmission of the infectious agent does not depend on the presence of infectious humans. Examples of these include tetanus and rabies.

In other applications, ignoring herd immunity will have some effect on the estimated cost-effectiveness of the program. For diseases that are more benign the older the age at infection (or indeed the severity of which is independent of the age at infection), ignoring herd immunity will underestimate the ben efits of widespread vaccination. Indeed, such a program would more than proportionally reduce the incidence of infection and shift the average age at infection to ages at which the disease is less (or equally) severe. Examples of such programs include childhood pertussis vaccination, universal $H B V$ vaccination and universal $H$ ib vaccination. When the evaluation of such vaccination programs yields favorable results, the analyst can assume that it will be even more favorable in reality. Hence, the associated simplification will not have changed the recommended decision. However, if the results are moderately unfavorable, the information provided by the analysis will only be of limited value to the decision-maker.

\section{When static models are inappropriate}

Some infections, however, cause more severe disease the older the age at infection. Hence it is important to assess whether the net effect of herd immunity is positive or negative. In the extreme case, at intermediate levels of vaccination coverage, vaccination programs could, for some diseases, cause more harm than good. Examples of these include childhood VZV and rubella vaccination. Clearly, before such programs are initiated it is vital to have a reliable estimate of future vaccination uptake, an issue we consider in more detail below. The use of static models to evaluate these programs can only be justified if a sufficiently high level of vaccination coverage can be attained during the firs tyear of the program and can be maintained at such high levels (new vaccination programs that are most likely to meet this requirement are those that are introduced immediately as an extra antigen in a combined vaccine, so that no additional injections are required and the coverage of other important vaccination programs is not endangered (e.g., the addition of varicella to the measles-mumps-rubella vaccine). At high vaccination coverage, the shift in the average age at infection would still occur but the number of cases would decrease in all age groups, including the older age groups. This means that the shift in the age at infection would not cause a greater burden of disease, even in the age groups that are at risk of more severe disease if infected. Immunity due to natural infection would then simply be replaced by vaccine-induced immunity in newly introduced susceptible persons. If, in a static model, vaccine protection is assumed to be of limited duration (or waning with time), a shift in the age at infection will also be generated by the model. However, it will not capture the complete shift observed in reality or produced by dynamic modeling. The informative value of the analysis would be very limited if results turn out to be moderately favorable (or moderately unfavorable) and, if instantaneously high vaccination coverage cannot be assumed, a dynamic model would be necessary to make a relevant analysis.

Dynamic models are also necessary to analyze programs targeted at core transmitter groups of a virus. Immunizing such groups would have complex nonproportional effects on the propagation of the virus in the population. Vaccination of a limited number of targeted people could then have a substantial impact on the epidemiology of infection, which cannot be described by static models. Examples of such interventions include targeted vaccination against blood-borne and sexually transmitted diseases of intravenous drug users and people with high sexual partner change in areas where the main routes of transmission are needle sharing and sexual intercourse (e.g., HIV and hepatitis B).

Cohort models are likely to be most accurate for the first cohort to be vaccinated and less and less so for further cohorts. This implies that, even more than for dynamic models, repeated economic evaluations are required as the epidemiological (and economic) parameters change after a program is implemented. Currently, there is very little attention being paid to such kind of analyses, as the tendency is to take a decision and not look back.

\section{No model at all?}

Empirical studies analyzing the costs and effects associated with important changes in vaccination policy would be very helpful in validating earlier predictions of modeling studies. To our knowledge such studies are currently lacking. A problem in a post hoc analysis is that it may not be clear which costs (for instance vaccination costs from 1996-1999) can be attributed to which benefits (reduced number of cases from 1996-1999?). Empirical cost studies alongside vaccine trials are slightly less rare. Their main disadvantage is that they are confined to the trial in terms of participants and duration. For many vaccines, this approach would provide very interesting, though insufficient information, as a trial does not capture all the costs and effects vaccination would have in the real world, particularly those that occur beyond the duration of the trial. Furthermore, it is unlikely that all options are explored in a trial and a trialanalysis takes more money and time than a model-based analysis would. Cost studies alongside vaccine trials have been published for PCV 7 [16] and PSV [17] vaccination. 


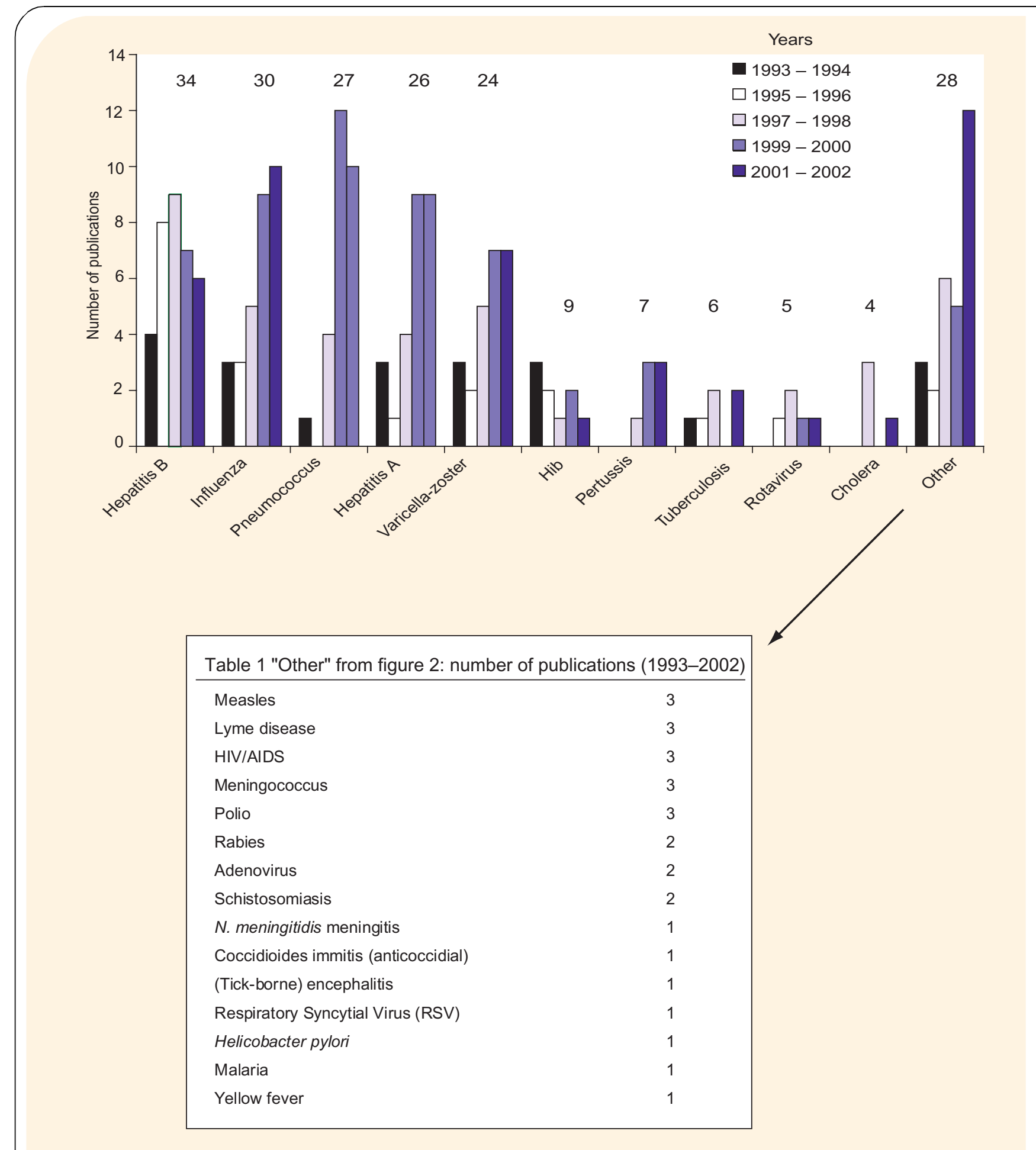

Figure 2. Average biannual number of publications related to economic evaluation of specific vaccines identified by searching We b of Science (1993-2002). Total number of publications per disease given on top of bars.

\section{New developments in modeling}

A current problem with dynamic models is that they have to rely on some core estimate expressing how a particular pathogen is transmitted in the population, based on the pathogen's characteristics (infectivity) and the population characteristics (receptiveness and social behavior). This estimate is typically formulated as a matrix that expresses which subgroups (usually based on age) acquire infection from which other subgroups. 
Until now these 'Who Acquires Infection From Whom' (WAIFW) matrices were usually (particularly for infections that can spread in a nonsexual way) based on epidemiological data only (albeit combined with intuition and common sense). Essentially this implied that somestructure was imposed on the matrix, without knowledge of its validity. Important future research would document social contact patterns (based on surveys) to inform this. In other words, the 'who mixes with whom' and 'who lives with whom' information could be used as a Bayesian prior to reduce arbitrariness in the estimate of the WAIFW matrix. There are only a few (pilot) studies that have attempted to document social mixing patterns related to the spread of infectious disease [18].

O ne of the most important parameters in infectious disease modeling is the force of infection. Another recent development is the application of flexible statistical and mathematical models to estimate the force of infection from prevalence and incidence data $[19,20]$. Further advances in statistical methods can be expected to improve the estimates of the force of infection and the WAIFW matrix.

Some of the most recent vaccines have including the expensive intranasal influenza (ca. US 40-50 per dose) and pneumococcal conjugate vaccine (ca. U $\$ \$ 30-40$ per dose) present particular challenges for epidemiological modeling. Widespread use of these vaccines is likely to exhibit complex indirect effects, impacting on herd immunity, antimicrobial resistance and circulating serotypes (whereby vaccine serotypes are replaced by nonvaccine serotypes ('serotype replacement'), vaccine serotypes may offer indirect protection against nonvaccine serotypes ('cross-reactivity') and the vaccine impact on disease burden may vary with time). Taking account of all these factors would require very large studies to gather data, some of which may only be collected after vaccination actually starts.

In future, dynamic modeling should become the standard for EEV P. In a dynamic model, even more than in a static model, the predicted effectiveness and cost-effectiveness of the program may be highly dependent on vaccination coverage (cost-effectiveness and cost-benefit ratios obtained through a static model are generally independent of assumed coverage [6]). Predicting vaccination coverage accurately is therefore important for EEVP, as well as for vaccine policy making in general. For some programs, good data on participation rates exist but for new vaccines this is not the case and in many countries existing programs may not offer a sufficient basis for predicting the uptake of new vaccines. Furthermore, in existing immunization programs, changes to program structure or incentives can lead to changes in uptake. Understanding this will allow policymakers to design programs so as to optimize immunization rates.

\section{Estimating \& valuing health outcomes \\ Deaths prevented \& life-years gained}

Estimates of age and time of deaths are usually generated by modeling (through case fatality ratios from empirical data). In order to estimate the loss of life-years from mortality data, life-expectancy by age must be known. For a number of infections, some people are at a greater risk of severe disease and mortality than others because of their overall health status (e.g., pneumococcal and influenza infections in the elderly). People dying from these diseases are generally in poorer health than average and would also have a lower life-expectancy than average, at a given age. Failing to take the diseasespecific aspects of life-expectancy into account may thus lead to overestimates of the benefits of the program.

$\mathrm{O} n$ the other hand, long-term projections of life-expectancy may be inaccurate because current overall mortality data are used to estimate the life-expectancy of people in the future, while life-expectancy is increasing with time. For instance, in western Europe the average life-expectancy at birth was about 40 years at the beginning of the 20th century and about 75 years at the end of it. Therefore, it seems that the benefits of interventions with long-term effectiveness may be systematically underestimated in this respect. One solution could be to apply a growth rate to the current estimates of life-expectancy but at present it seems very speculative to determine such a growth rate.

\section{Quality-adjusted life-years gained}

Q uality-adjusted life-years (Q ALYS) are the most widely used outcome measures that combine morbidity with mortality [21].

A change in the quality of life due to an intervention can generally be written as:

$$
\Delta \mathrm{Q} A \mathrm{LY}=\mathrm{L} \cdot \Delta \mathrm{q}+\mathrm{q} \cdot \Delta \mathrm{L}
$$

The first term refers to quality of life variation $(\Delta q)$ during years that would have been lived without the intervention (L) and is an expression of pure quality of life improvement. The second term refers to the qual ity of life during years gained by the intervention $(\Delta \mathrm{L})$ and is therefore only relevant for life-saving interventions. The impact on the quality of life may be very important for some (i.e., many interventions are not life-saving and only have an impact on the quality of life), while it could be negligible for other interventions (i.e., interventions that predominantly extend the duration of life and not the quality of life). M ost vaccination programs seem to fall within the latter category. They predominantly extend life and often may only to a lesser degree have an impact on the quality of life, for a short period of time. For many vaccine-preventable childhood infections, the morbidity following natural infection is relatively short lived (i.e., days, weeks) and results in either recovery or death. H ence most of the health gain is obtained through the avoidance of mortality and the associated extension of life (i.e., years, decades). H owever, quality gains form an important benefit for vaccination programs aimed at infections leading frequently to long-lasting complications. In contrast, EEV Ps of programs targeted at people who al ready have a substantially reduced quality of life at the time of the intervention (many therapeutic interventions but also vaccination programs aimed at the elderly [such as influenza and pneumococcal vaccination]) will overestimate the health gains if quality of life aspects are ignored. 
Conceptual and methodological problems as well as the considerable work and costs required for a proper job, often lead analysts to ignore quality of life aspects. In a review of EEVPs conducted in April 2001, only 19 such studies were found [3]. Eight of these were on pneumococcal vaccination (of which five papers were from the same study but spread out over multiple papers by two groups of authors), three on hepatitis $\mathrm{A}$ vaccination (a rarely fatal disease), three on $\mathrm{H} \mathrm{ib}$ (a relatively rare infection with great potential impact on quality of life) and five more on various infections which can have an important impact on quality of life ( $\mathrm{N}$ eisseria meningitidis meningitis, HIV, respiratory syncytial virus, Lyme disease and varicella in adults). The earliest of these publications appeared in 1995 [3].

\section{Beyond QALYs}

H owever, there are situations where Q ALYs or other health status measures do not capture all benefits that may be relevant to consumers [22]. Cost-effectiveness and cost-utility analyses, in which health gains are typically expressed in these terms, may then not provide sufficient information for priority setting. In the case of immunization, individuals may value peace of mind from knowing they (or their children) have a reduced risk of illness. For instance, the anxiety caused by epidemic cycles of polio and more recently, anxiety related to meningococcal infections and SARS in industrialized countries, and HIV and sleeping sickness in developing countries illustrate this point. Consumers may also value the avoidance of the disruption to normal daily life, including time away from work caused by illness or caring for a sick child. N egative effects may occur due to the vaccination program itself. Individuals may be anxious about the outcome of vaccination due to the chance of adverse events, which may in some cases be severe. Since all potential vaccinees (and/or their parents) may incur such 'anxiety costs', the sum of all these costs may be substantial for some programs (particularly when there is rumor about al leged adverse events, as for instance currently in the UK with MMR). These various aspects (sometimes described as intangible costs and benefits) can be taken into account by using cost-ben efit analysis, in which all health gains are valued in monetary terms.

Therefore, there is an increasing interest in the development of willingness-to-pay measures to value the outcomes of an intervention. In a study in N orth California, 1657 parents who had their 1 to 8 month old infant vaccinated during the previous 14 days were asked for their willingness to pay (WTP [i.e., stated and revealed preference methods]) for reducing the number of childhood injections per visit. The median W TP was $\$ 25$ for a reduction in the number of injections from 4 to 3 , or from 3 to 2 , whereas it was $\$ 50$ for a reduction from 2 to 1 . Parents whose child showed clinical adverse events indicated that the median WTP to avoid these would be $\$ 50$. The very wide range for each of these estimates (minimally \$0-1000) may indicate that people have different perceptions of the risks involved, have different attitudes towards risk and generally have difficulty in quantifying their hypothetical WTP. Nonetheless the median value exceeded the average estimated costs of adverse events ( $\$ 7.7$ per vaccinated child, including work loss to the parents $(53 \%)$ in the total study population [23]. Therefore, it seems that the intangible costs of a vaccination program may not be negligible. Clearly, more studies are needed to substantiate these results and to determine whether WTP methods can appropriately measure these costs. However, it remains questionable whether healthy persons, who typically answer WTP questions, can imagine what it is like to be in various states of illhealth; or whether persons not used to paying the full costs of healthcare will provide accurate estimates of benefit [24,25].

\section{Discrete choice experiments to understand consumer preferences}

The assessment of a monetary value for the benefits of vaccination is part of the broader topic of understanding consumer preferences. This can be addressed by means of discrete choice experiments (DCEs). There are two components to DCES, the use of experiments to generate a data set on preferences for health programs and the use of discrete choice analysis to model these preferences. The approach was developed in transport economics [26] and marketing [27], has been adopted in environmental economics [28] and more recently in health economics [29]. More detail on theory and methods can be found in Louviere and colleagues [30]. The underlying assumption is that individuals will choose, from among the available options, the one that is best for them, i.e., the one that maximizes their utility. Furthermore, any product, or in this case, immunization program, can be described in terms of its attributes or characteristics. In the case of vaccines, such attributes might include the severity of the disease in question, the side effects from immunization, whether it requires a separate injection or can be combined into a multivalent vaccine, whether it is available at clinics, or requires a special doctor visit. The analyst can then describe a number of proposed programs in terms of the levels of these different attributes. The experiment presents respondents with a number of hypothetical choices described in this way. In choosing their preferred option, individuals will make trade-offs across the levels of the different attributes, so as to maximize their utility given each choice set. In the case of vaccination, the resultant models can provide an estimate of the probability that individuals will be immunized, given the design of the program and the attributes of the vaccine. Interpretation is not completely straightforward as the parameter estimates are confounded with variance of the error term; nonetheless the results can be used to design immunization programs to optimize uptake. In particular, where data from a discrete choice experiment can be combined with revealed preference (market) data, it is possible to identify the differences in the scale of the parameters that results from differences in unobserved variability between samples [31]. Adjusting for relative scale yields parameter estimates that will allow for accurate prediction of uptake. While 
this approach to forecasting demand is quite common in other areas of economics and marketing, there are very few examples of health program applications [32]. The models can also be used to identify effective strategies for different groups within the target population.

The inclusion of price as an attribute in the DCE will generate data about respondents' tradeoffs between price and other aspects of the program. This can be interpreted as W TP for improvements in the vaccine and/or program design and thus provides a money measure of the value of additional benefits. Monetary valuations of benefits are required for any cost-benefit analysis and, as noted above, there is increasing interest in using cost- benefit analysis to encompass an appropriate range of benefits of healthcare and public health programs. This form of analysis has been limited in health applications because there is very limited price information for health programs, even in systems where some consumer payments are common. There are other approaches to obtaining W T P data, such as contingent valuation. $\mathrm{H}$ owever, there are a number of advantages in using DCEs. First, respondents are required to make choices and are thus forced into making trade-offs across attributes, thus reducing the tendency to accept the program that is offered. Second, although respondents may be unfamiliar with new vaccines or program changes, the description of options in terms of attributes can provide information in a way that respondents can relate to programs with which they are familiar. Third, the use of hypothetical examples allows a range of attributes and levels to be considered, providing a much richer set of information about the possible policy options.

\section{Cost estimation}

\section{Marginal intervention costs}

In EEVP, the intervention costs often have a great impact on the cost-effectiveness because they arise in the present to prevent disease in the future (which will be scaled down by discounting, see below). The costs of adding a particular vaccine to the existing range of vaccines depends on how well the schedule of the new vaccine fits in with the other schedules, whether specific precautions need to be taken, whether potential vaccinees need to be screened prior to vaccination or whether a specific target group is envisaged. For example, the administration costs of adding varicella vaccination to the existing M M R vaccination is likely to be lower than the current average administration costs of either measles, mumps or rubella vaccination because it only requires one extra injection at the same time as the existing injection of M M R vaccine. The costs that are most heavily affected by adding a new vaccine to the existing program are the variable costs of the program (e.g., time spent per vaccinee, number of vaccines bought), whereas the influence on the fixed intervention costs (e.g., buildings, general equipment) is usually small, or even negligible (unless a new vaccine requires a substantially different infrastructure in terms of storage and transport). An example illustrating this point is provided by $\mathrm{H}$ all and colleagues who examined the immunization program in $\mathrm{T}$ he $\mathrm{G}$ ambia (more recently these results were confirmed by a similar analysis in Addis Ababa [34]) [33]. They found that the additional costs of adding hepatitis $B$ vaccine to the existing Expanded Program on Immunization (EPI) vaccines (measles, polio, Bacille $C$ al mette- $G$ uérin [BC G], pertussis, diphtheria, tetanus), would be $94 \%$ recurrent costs (of which $87 \%$ for purchasing hepatitis B vaccine). $\mathrm{N}$ evertheless, nonrecurrent set-up costs of a new vaccination (consisting mainly of information campaigns of both people in the target group and vaccine administrators) could be substantial for some vaccines and countries (an example for this is provided by Scuffham and colleagues for VZV vaccination in Australia [35]). In some evaluations it may be difficult to disentangle the part of the vaccination costs that are related to the vaccination program under review. For instance, the optimization of the coverage of M MR vaccination in European countries aims in the first place to avoid measles outbreaks in the future, in the second place to avoid rubella infection in pregnant women and in the third place to avoid additional cases of mumps. In an analysis of measles vaccination in countries where M M R or $M R$ vaccines are most widely used, there might be problems with the allocation of costs to individual components in the combination vaccines. A subjective judgement may be necessary to decide how the marginal vaccination costs should be divided across the various antigens. For instance, the reason for increasing coverage of M M R vaccination from $80 \%$ to $95 \%$ in view of a measles elimination program, could be judged to be $50 \%$ due to measles, $30 \%$ due to rubella and $20 \%$ due to mumps [36,37].

A problem of a more practical nature and only indirectly related to economic evaluation, is for program managers to choose one combination vaccine among the various vaccines on offer, all with varying schedules, prices and antigens. Linear programming could be helpful in selecting the package of vaccines that best fits the local organizational structure and budget constraints [38]. It should be noted that these techniques consider costs of purchase and administration, schedule and other characteristics of the vaccination but not the population benefits of a program and therefore cannot serve as a substitute for economic evaluation.

\section{Indirect costs}

The terms indirect costs and productivity costs have been used interchangeably [39]. In a more appropriately refined definition, indirect costs encompass both costs of lost time (either productive time or leisure time, or a combination of both) and costs that are unrelated to diseases affected by the intervention. An economic eval uation is al ways a partial analysis, based on a partial equilibrium. Although it is clear that any change in economic activity (such as the investment in a vaccination program) will cause many ripples throughout the economy, the analyst assumes that we can artificially 'seal off' an area of the economy, by postulating that all else remains 
constant. In practice, the analyst will have to find a balance between what is theoretically correct and technically possible, without undermining the credibility of the findings. It is therefore not surprising that there is some disagreement on which indirect costs to include in an economic evaluation.

Indirect cost estimates could be very influential for some vaccination programs, particularly so for rarely fatal infections that cause the infected or their parents to incur time costs. For instance, universal childhood VZV vaccination is relatively cost-ineffective for the healthcare payer in terms of preventing mortality and serious disease. However, from a societal point of view varicella vaccination results in substantial net savings, thanks to avoided time losses of parents and other caretakers [4]. H ence the inclusion of time losses solely changes the decision. It is therefore unfortunate that these costs, by lack of reliable and widely accepted methodologies are usually not deemed credible. In view of this, there seems to be an important role for threshold analysis on the cut-off value per lost unit of time (see for example Beutels and colleagues [VZV] [40], and Beutels and Gay [measles] [37]).

There is an ongoing debate about the appropriate methods to estimate the costs of time losses, due to both morbidity and mortality. The main contenders are the human capital, friction cost and WTP methods.

Indirect costs of other diseases acquired during the prolonged lifetime (i.e., 'future costs' also called unrelated costs) are usually not taken into account because they are difficult to estimate and because it is unclear whether it is methodologically sound to include them at all [41]. Vaccination programs that are most prone to be influenced by the inclusion of unrelated healthcare costs are those aimed at persons at increased risk of comorbidity, such as pneumococcal and influenza vaccination targeted at the elderly. For these target groups, the time span between intervention and competing morbidity is relatively short and therefore the current practice of omitting unrelated healthcare costs creates an added bias in favor of these vaccination programs versus other ones (similar to the bias if gains in life expectancy are based on average life expectancy). For illustrations of this see Sisk and colleagues [42], and M eltzer [43]. As well as unrelated healthcare costs, there are also unrelated nonhealthcare costs, usually considered as consumption net of earnings which will be a net cost for the elderly but a net contribution to the economy for young adults. When these are included, the effect of preventing deaths in young adults may substantially lower (i.e., improve) the cost-effectiveness ratio [44]. When comparing cure to prevention, however, the exclusion of unrelated costs (which is current practice) would mostly seem to create a bias in favor of cure as those people al ready sick generally have the highest immediate additional healthcare costs. It seems therefore that current practice of economic evaluation should be changed to take into account future unrelated costs, if it is to inform policy in a more consistent way. The impact this would have on equity, however, would need to be assessed separately.

\section{Discounting}

Discounting is a technique in economic evaluation by which future events (e.g., costs and health outcomes) are valued less the further in the future they arise. Although there is general agreement on the discounting of costs, the arguments for discounting non-monetised health outcomes are contradictory. Empirical evidence to date is limited to individuals' time preferences, which in general would seem to be positive for health (demanding a positive discount rate). H owever, choosing an appropriate expression of social time preference is essentially a normative issue that can only be resolved explicitly through social debate or by eliciting representative social preferences. Discounting costs without discounting benefits leads to the paradoxical situation that any eradication program will yield infinite benefits, implying that all resources should be spent on research on eradicable diseases. H owever, the arguments for an equal discount rate for both costs and health effects are essential ly negative, constructed to avoid logical inconsistencies. The underpinnings and relevance of these are questionable, so that a lower discount rate for health effects than for costs is not necessarily wrong. Additionally the choice of the discount model (i.e., stationary vs nonstationary models) and of the appropriate rate of discount seems to be based primarily on convention. Further empirical research is needed to strengthen or to change the basis for this conventional approach.

Prevention programs in general and some vaccination programs, such as hepatitis B vaccination, in particular, are very sensitive to discounting (of costs as well as health effects). N onetheless, this is no cause for treating preventive interventions differently from curative interventions.

Some of the other key issues mentioned above can be influenced by discounting. For instance the impact of ignoring unrelated cost, quality of life or herd immunity would all become substantially greater at lower discount rates or by using nonstationary discount models.

\section{Conclusion}

In this paper, we summarized a range of specific issues that present methodological challenges to EEVP. We noted that despite the ever increasing popularity of economic evaluations of vaccines, there are still important areas that need more research. In particular, we discussed the choice of mathematical model and appropriate estimation of key epidemiological parameters, the valuing of health outcome measures, the assessment of intervention and indirect costs and the choice of discount model in this specific context. Furthermore, we highlighted the important contribution of DCE to provide better estimates of willingness to pay and vaccine uptake, two key elements for estimating the ben efits of a vaccination program.

\section{Five-year view}

$N$ ew developments in vaccines will lead to more multivalent vaccines and to more specific vaccines targeted at particular susceptible groups. As the pressure on healthcare budgets is unlikely to lessen, we expect the role of economic evaluation (EE) in this 
area to gain importance. As the new vaccines on the agenda for policy-making in weal thy countries prevent 'low profile' illnesses and are likely to be judged borderline cost-effective, decisions regarding vaccination are becoming less straightforward.

The shortcomings of EE in this field, as outlined above, will receive more attention. In particular, we expect that epidemiological modeling will improve as more multidisciplinary research, combining expertise in epidemiology, mathematical modeling and economics is initiated. Increased understanding of the process of disease transmission and the role and infectivity of different population subgroups, will provide the basis for a more complex model structure. $\mathrm{N}$ ew models will not only demonstrate the role of different subgroups and social structures in disease transmission but will also show the distribution of vaccine effects and costs over subgroups. M ore investigation of the behavior of individuals and groups in response to new vaccine programs will provide more accurate estimates of uptake rates.

To date, EEV Ps have approached the measurement of benefits as fairly simple health outcomes, such as the number of cases prevented, or the number of life-years gained. We can expect to see a more sophisticated approach develop over the next 5 years. Life expectancy measures may take into account the changing life expectancy over time and differences in subgroups. D evelopments in the measurement and valuation of quality of life will be transferred to EEVPS. The other aspects, both positive and negative, of vaccination programs and infectious disease, the intangible benefits and costs, will be recognized and explored. These will be incorporated into EEVP results, using new measures based on willingness to pay and estimated using the sophisticated approaches of DCEs.

On the cost side, there will be concomitant developments in modeling program and treatment costs to provide more valid estimates of the marginal opportunity cost. More debate on the role and significance of indirect costs is to be expected. Similarly on the issue of discounting, some empirical exploration of the social values for time preference and more investigation of the significance of the choice of different rates is expected. The solution to these issues will largely depend on the evolution of health economic evaluation in general, as at present there seems to be a tendency to advocate different approaches to valuing health outcomes and discounting.

\section{Expertopinion}

Evaluations of vaccination programs are usually based on static models, which essentially assume that infectious diseases are not infectious. Though this approach can be acceptable for particular vaccination strategies and target groups, it is often inappropriate. Dynamic models, which take the communicable properties of vaccine preventable diseases into account, should be much more widely used and eventually become standard practice for the evaluation of vaccination programs. Improved methodology in estimating the force of infection (a required estimate in both static and dynamic models), and new initiatives for empirical data collection on social interaction between different subgroups in populations (to inform the 'W ho Acquires Infection From Whom' matrix, a required estimate in dynamic models only), should further improve infectious disease models.

There is a need in EEVPs for more consistent incorporation of quality adjusted survival gains, which would improve the estimate and valuation of health outcomes, and hence the comparability between programs. Furthermore, there is a need for a strong consensus on discounting, in order to retain the credibility of economic evaluation of prevention. Indirect and intangible costs and benefits are difficult to estimate, and often ignored. These may nonethel ess be substantial for infectious diseases and vaccination programs, and decision making could be misled if they are not accounted for. The estimate of marginal intervention costs also presents specific difficulties, especially when evaluating one component in a multivalent vaccine. Joint evaluations of all components of multival ent vaccines are therefore preferable, and we should strive to make it standard practice in the future.

D iscrete choice event modelling provides an interesting alternative to predict uptake rates, an important element in estimating both the costs and effects of a program, and could also be useful to estimate willingness to pay for vaccination programs.

\section{Key issues}

- Economic evaluation does not appear to have been used consistently in policy making for immunization.

- Dynamic modelling, taking the communicable properties of vaccine preventable diseases into account, should be more widely used and eventually become standard practice.

- New empirical studies on social interaction and new methods to estimate the force of infection will improve predictions from infectious disease modelling.

- There is a need for a strong consensus on discounting in order to retain the credibility of economic evaluation.

- Potentially substantial costs and benefits are routinely ignored, due to problems of estimation and credibility.

- Discrete choice event modelling could offer an alternative to predict uptake rates and estimate the willingness to pay for vaccination. 


\section{References}

Papers of special note have been highlighted as: - of interest

- of considerable interest

1 Drummond M. Cost-effectiveness guidelines for reimbursement of pharmaceuticals: is economic evaluation ready for its enhanced status? Health Econ. 1, 85-92 (1992).

- BeutelsP, Edmunds WJ, Antoñanzas F et al. Economic evaluation of vaccination programmes: a consensus statement focusing on viral hepatitis.

Pharmacoeconomics 20(1), 1-7 (2002).

- A consensus statement on many of the issues in this paper by a group of economists with considerable expertise on vaccination.

3 BeutelsP. Economic evaluation of vaccination programmesin humans a methodological exploration with applications to hepatitis $B$, varicella-zoster, meases, pertussis, hepatitisA and pneumococcal vacanation. University of Antwerp, Antwerp, Belgium (2002).

${ }_{4}$ Thiry N, BeutelsP, Van DammeP, Van Doorsl aer $E$. Economic evaluations of varicella vaccination programmes: review of the literature. Pharmacoeconomics 21(1), 13-38 (2003).

- Beutels P, PostmaM J. Economic evaluations of adult pneumococcal vaccination: a review of the literature. Expert Rev. Pharmacoeconomics Outcomes Res. 1(1), 47-58 (2001).

-6 BeutelsP. Economic evaluations of hepatitis $B$ immunization: a global review of recent studies (1994-2000). Health Econ. 10(8), 751-774 (2001).

7 Postma MJ, Jansema P, van Genugten M L et al. Pharmacoeconomics of influenza vaccination for heal thy working adults: reviewing the avai lable evidence. D rugs 62(7), 1013-1024 (2002).

${ }_{8}$ Rosenthal P. Cost-effectiveness of hepatitis $A$ vaccination in children, adolescents, and adults. Hepatology 37(1), 44-51 (2003).

$\rightarrow$ McKie J, Richardson J. The rule of rescue. Soc. Sci. M ed. 56, 2407-2419 (2003).

10 Anderson RM, May RM . InfectiousD iseases of H umans- Dynamics and Control. Oxford University Press, Oxford, UK (1991).

11 Daley DJ, Gani J. Epidemic modelling: an introduction. Cambridge University Press, Cambridge, UK (1999).

-12 Keling MJ, Grenfell BT. Disease extinction and community size: modeling $\nabla_{24}$ the persistence of measles. Science 275(5296), 65-67 (1997). $\checkmark 13$ Lieu TA, Cochi SL, Black SB et al. Costeffectiveness of a routine varicella vaccination program for US children. JAM A 271(5), 375-381 (1994).

${ }_{14}$ Halloran ME, Watelet L, Struchiner CJ. Epidemiologic effects of vaccines with complex direct effect in an age structured population. M ath. Biosci. 121(2), 193-225 (1994).

-15 EdmundsWJ, M edley GF, N okesDJ. Evaluating the cost-effectiveness of vaccination programmes: a dynamic perspective. Stat. M ed. 18, 3263-3282 (1999).

-16 Ray GT, Butler JC, Black SB, Shinefield $\mathrm{HR}$ \&t al. O bserved costs and health care use of children in a randomized controlled trial of pneumococcal conjugatevaccine. Pediatr. Infect. D is. J. 21(5), 361-365 (2002).

Nichol KL, Baken L, Wuorenma J, Nelson A. Thehealth and economic benefits associated with pneumococcal vaccination of elderly persons with chronic lung disease. Arch. Intern. M ed. 159(20), 2437-2442 (1999).

18 WallingaJ, Edmunds WJ, Kretzschmar M. Perspective: human contact patterns and the spread of airborne infectious diseases. Trends M icrobiol. 7(9), 372-377 (1999).

19 Shkedy Z, Aerts M, M olenberghs G, BeutelsP, Van D ammeP. Modeling the force of infection using monotone local polynomials. Appl. Stat. (2003) (In Press). van Boven M, de M elker HE, Schellekens $J F$, K retzschmar M. Waning immunity and sub-cinical infection in an epidemic model: implications for pertussisin The Netherlands. M ath. Biosci. 164(2), 161-182 (2000).

- 21 Gold M R, Stevenson D, Fryback DG. HALYS and QALYS and DALYS, Oh My: similarities and differences in summary measures of population Health. Ann. Rev. Public Health 23, 115-134 (2002).

${ }_{22}$ Dolan P. Utilitarianism and the measurement and aggregation of qualityadjusted lifeyears. Health CareAnal. 9(1), 65-76 (2001).

23 Lieu TA, Black SB, Ray GT, M artin KE, Shinefield HR, Weniger BG. The hidden costs of infant vaccination. Vaccine 19(1), 33-41 (2000).

- Costing study illustrating that the intangible costs of vaccination may be substantial.

4 Olsen JA, Smith RD. Theory versus practice: a review of 'willingness-to-pay' in health and health care. Health Econ. 10(1), 39-52 (2001).
- An extensive review of willingness-to-pay studies in health care, it's theoretical basis visa-visQ ALYsdiscussing to which extent practice corresponds with theory.

${ }_{25}$ Smith RD. Construction of the contingent valuation market in health care: a critical assessment. $H$ ealth Econ. 12(8), 609-628 (2003).

- 26 Hensher D. Stated preference analysis of travel choices: the state of practice. Transportation, 21, 107-133 (1994).

27 Ben-Akiva M, Boccara B. Discrete choice models with latent choice sets. Int. J. Res M arketing 12, 9-24 (1995).

-28 Adamowicz W, LouviereJ, Williams M. Combining revealed and stated preference methods for valuing environmental amenities. J. Environ. M anage. Econ. 26, 271-292 (1994).

29 Viney R, Lancsar E, Louviere J. D iscrete choice experiments to measure consumer preferences for health and heal thcare Expert Rev. Pharmacoeconomics Outcomes Res. 2(4), 319-326 (2002).

30 LouviereJJ, H ensher D A, Swait JD. Stated Choice $M$ ethods analysis and applications. Cambridge University Press, Cambridge UK (2000).

$\nabla_{31}$ Swait J, LouviereJ. Therole of the scale parameter in the estimation of estimation and comparison of multinomial logit models. J. M arketing Res. 30, 305-314 (1993).

- $32 \mathrm{H}$ all J, Kenny P, King M et al. U sing stated preference discrete choice modelling to evaluate the introduction of varicella vaccination. Health Econ. 11, 457-465 (2002).

- The only application of discrete choice event modelling in the fiedd of vaccination.

$33 \mathrm{H}$ all AJ, Robertson RL, Crivelli PE et al. Cost-effectiveness of hepatitis $B$ vaccinein The Gambia. Trans. R. Soc. Trop. M ed. Hyg 87, 333-336 (1993).

- 34 Edmunds WJ, Dejene A, M ekonnen Y, $\mathrm{H}$ aile $M$ et al. The cost of integrating hepatitis $B$ virus vaccineinto national immunization programmes: a casestudy from Addis Ababa. Health Policy Plann. 15(4), 408-416 (2000).

35 Scuffham PA, Lowin AV, Burgess MA.The cost-effectiveness of varicella vaccine programs for Australia. Vaccine 18(5-6), 407-415 (1999).

- 36 Beutels P, Van DammeP, Van Casteren V, Gay N et al. The difficult quest for data on 'vanishing' vaccine-preventableinfections in Europe: the case of measles in Flanders (Belgium).Vaccine20(29-30), 3551-3559 (2002). 
-37 Beutels Ph, Gay NJ. Economic evaluation of options for measles vaccination strategy in a hypothetical western European country. Epidemid. Infect. 130(2), 273-283 (2003).

- A recent example of the use of a dynamc epidemiological model for costeffectiveness and cost- benefit analysis.

- 38 Weniger BG, Chen RT, Jacobson SH, Sewell EC et al. Addressing the challenges to immunization practicewith an economic al gorithm for vaccine selection. Vaccine 16(19), 1885-1897 (1998).

39 LuceBR, M anning WG, Siegel JE, Lipscomb J. Estimating costs in costeffectiveness analysis. In: Cost-effectiveness in Health and M edicine. Gold M R, Siegel $J E$, Russel LB, Weinstein MC (Eds). Oxford University Press, NY, USA 176-213 (1996).

40 Beutels Ph, Clara R, TormansG, Van D oorslaer $E$, Van DammeP. Costs and benefits of routine varicella vaccination in German children, J. Infect. Dis. 174(Suppl. 3), S335-341 (1996).
41 Garber AM, Weinstein M C, Torrance GW, ${ }_{45}$ Kamlet MS. Theoretical foundations of cost-effectiveness analysis. In: Costeffectiveness in $H$ ealth and $M$ edicine. Gold $M R$, Siegel JE, Russe LB, Weinstein MC (Eds). O xford University Press, NY, USA 25-53 (1996).

42 Sisk JE, M oskowitz AJ, Whang W. et al. C ost-effectiveness of vaccination against pneumococcal bacteremia among elderly people JAM A 278(16), 1333-1339 (1997).

${ }_{43}$ M eltzer D. Accounting for future costsin medical cost-effectiveness analysis. J. H ealth Econ. 16(1), 33-64 (1997).

${ }_{44}$ M eltzer D, Egleston B, Stoffel D, D asbach E. Effect of future costs on costeffectiveness of medical interventions among young adults: the example of intensive therapy for type 1 diabetes mellitus. M ed. Care 38(6), 679-685 (2000).
Brisson M , Edmunds WJ. Economic evaluation of vaccination programs: the impact of herd immunity. M ed. Decis M aking 23(1), 76-82 (2003).

-. A well-organized overview outlining the various issues of dynamic versus static modelling, with illustrations on cost- effectiveness impact

\section{Affiliations}

- Philippe Beutels, National Center for Immunisation Research and Surveillance, University of Sydney and Center for $\mathrm{H}$ ealth Economics Research and Evaluation, U niversity of Technology at Sydney, Australia; Centre for the Evaluation of Vaccination, U niversity of Antwerp, Belgium

- Eddy Van D oorslaer, D epartment of $\mathrm{H}$ ealth Policy and $M$ anagement, Erasmus $U$ niversity M edical Center, Rotterdam, N etherlands

- PierreVan Damme, Center for the Evaluation of Vaccination, U niversity of Antwerp, Belgium

- Jane H all, Centre for H ealth Economics Research and Evaluation, U niversity of Technology at Sydney. 